\title{
Another type of Mann iterative scheme for two mappings in a complete geodesic space
}

\author{
Yasunori Kimura and Koichi Nakagawa*
}

"Correspondence:

6513009n@nc.toho-u.ac.jp

Department of Information Science,

Toho University, Miyama, Funabashi,

Chiba 274-8510, Japan

\begin{abstract}
In this paper, we show a $\Delta$-convergence theorem for a Mann iteration procedure in a complete geodesic space with two quasinonexpansive and $\Delta$-demiclosed mappings. The proposed method is different from known procedures with respect to the order of taking the convex combination.
\end{abstract}

\section{Introduction}

The fixed point approximation has been studied in a variety of ways and its results are useful for the other studies. In 1953, Mann [1] introduced an iteration procedure for approximating fixed points of a nonexpansive mapping $T$ in a Hilbert space. Later, Reich [2] discussed this iteration procedure in a uniformly convex Banach space whose norm is Fréchet differentiable. In 1998, Takahashi and Tamura [3] considered an iteration procedure with two nonexpansive mappings and obtained weak convergence theorems for this procedure in a uniformly convex Banach space which satisfies Opial's condition or whose norm is Fréchet differentiable. On the other hand, in 2008, Dhompongsa and Panyanak [4] proved the following theorem.

Theorem 1.1 Let $C$ be a bounded closed convex subset of a complete $\mathrm{CAT}(0)$ space and $T: C \rightarrow C$ a nonexpansive mapping. For any initial point $x_{0}$ in $C$, define the Mann iterative sequence $\left\{x_{n}\right\}$ by

$$
x_{n+1}=\left(1-t_{n}\right) x_{n} \oplus t_{n} T x_{n}, \quad n=0,1,2, \ldots,
$$

where $\left\{t_{n}\right\}$ is a sequence in $[0,1]$, with the restrictions that $\sum_{n=0}^{\infty} t_{n}$ diverges and $\lim \sup _{n \rightarrow \infty} t_{n}<1$. Then $\left\{x_{n}\right\} \Delta$-converges to a fixed point of $T$.

Further, in a CAT(1) space, Kimura et al. [5] proved the $\Delta$-convergence theorem for a family of nonexpansive mappings including the following scheme:

$$
x_{n+1}=\left(1-\alpha_{n}\right) x_{n} \oplus \alpha_{n}\left(\left(1-\beta_{n}\right) S x_{n} \oplus \beta_{n} T x_{n}\right) .
$$

In a Hilbert space $H$, the following equality holds for any $x, y, z \in H$ :

$$
\alpha x+(1-\alpha)(\beta y+(1-\beta) z)=\gamma(\delta x+(1-\delta) y)+(1-\gamma) z
$$


where $\alpha, \beta, \gamma, \delta \in] 0,1[$ such that $\alpha=\gamma \delta$ and $\beta=\gamma(1-\delta) /(1-\gamma \delta)$. However, in $\operatorname{CAT}(\kappa)$ spaces with $\kappa>0$, it does not hold in general, that is, the value of the convex combination taken twice depends on their order. Thus, the following formulas are different in general:

$$
\begin{aligned}
& x_{n+1}=\left(1-\alpha_{n}\right) x_{n} \oplus \alpha_{n}\left(\left(1-\beta_{n}\right) S x_{n} \oplus \beta_{n} T x_{n}\right), \\
& x_{n+1}=\left(1-\alpha_{n}\right)\left(\beta_{n} x_{n} \oplus\left(1-\beta_{n}\right) S x_{n}\right) \oplus \alpha_{n}\left(\left(1-\beta_{n}\right) x_{n} \oplus \beta_{n} T x_{n}\right) .
\end{aligned}
$$

In this paper, we show an analogous result to Theorem 1.1 using the iterative scheme (1) in a complete CAT(1) space with two quasinonexpansive and $\Delta$-demiclosed mappings. We also deal with the image recovery problem for two closed convex sets.

\section{Preliminaries}

Let $X$ be a metric space. For $x, y \in X$, a mapping $c:[0, l] \rightarrow X$ is said to be a geodesic if $c$ satisfies $c(0)=x, c(l)=y$ and $d(c(s), c(t))=|s-t|$ for all $s, t \in[0, l]$. An image $[x, y]$ of $c$ is called a geodesic segment joining $x$ and $y$. For $r>0, X$ is said to be an $r$-geodesic metric space if, for any $x, y \in X$ with $d(x, y)<r$, there exists a geodesic segment $[x, y]$. In particular, if a segment $[x, y]$ is unique for any $x, y \in X$ with $d(x, y)<r$, then $X$ is said to be a uniquely $r$-geodesic metric space. In what follows, we always assume $d(x, y)<\pi / 2$ for any $x, y \in X$. Thus, we say $X$ is a geodesic metric space instead of a $\pi / 2$-geodesic metric space. For the more general case, see [6].

Let $X$ be a uniquely geodesic metric space. A geodesic triangle is defined by $\triangle(x, y, z)=$ $[x, y] \cup[y, z] \cup[z, x]$. Let $M$ be the two-dimensional unit sphere in $\mathbb{R}^{3}$. For $\bar{x}, \bar{y}, \bar{z} \in M$, a triangle $\triangle(\bar{x}, \bar{y}, \bar{z}) \subset M$ is called a comparison triangle of $\triangle(x, y, z)$ if $d(x, y)=d_{M}(\bar{x}, \bar{y})$, $d(y, z)=d_{M}(\bar{y}, \bar{z}), d(z, x)=d_{M}(\bar{z}, \bar{x})$. Further, for any $x, y \in X$ and $\left.t \in\right] 0,1[$, if $z \in[x, y]$ satisfies $d(x, z)=(1-t) d(x, y)$ and $d(z, y)=t d(x, y)$, then $z$ is denoted by $z=t x \oplus(1-t) y$. A point $\bar{z} \in[\bar{x}, \bar{y}]$ is called a comparison point of $z \in[x, y]$ if $d(x, z)=d_{M}(\bar{x}, \bar{z}) . X$ is said to be a CAT(1) space if, for any $p, q \in \triangle(x, y, z) \subset X$ and its comparison points $\bar{p}, \bar{q} \in \Delta(\bar{x}, \bar{y}, \bar{z}) \subset M$, the inequality $d(p, q) \leq d_{M}(\bar{p}, \bar{q})$ holds.

Let $X$ be a geodesic metric space and $\left\{x_{n}\right\}$ a bounded sequence of $X$. For $x \in X$, we put $r\left(x,\left\{x_{n}\right\}\right)=\limsup _{n \rightarrow \infty} d\left(x, x_{n}\right)$. The asymptotic radius of $\left\{x_{n}\right\}$ is defined by $r\left(\left\{x_{n}\right\}\right)=$ $\inf _{x \in X} r\left(x,\left\{x_{n}\right\}\right)$. Further, the asymptotic center of $\left\{x_{n}\right\}$ is defined by $A C\left(\left\{x_{n}\right\}\right)=\{x \in X$ : $\left.r\left(x,\left\{x_{n}\right\}\right)=r\left(\left\{x_{n}\right\}\right)\right\}$. If, for any subsequences $\left\{x_{n_{k}}\right\}$ of $\left\{x_{n}\right\}, A C\left(\left\{x_{n_{k}}\right\}\right)=\left\{x_{0}\right\}$, i.e., their asymptotic center consists of the unique element $x_{0}$, then we say $\left\{x_{n}\right\} \Delta$-converges to $x_{0}$ and we denote it by $x_{n} \Delta x_{0}$.

Let $X$ be a metric space. A mapping $T: X \rightarrow X$ is said to be a nonexpansive if $T$ satisfies $d(T x, T y) \leq d(x, y)$ for any $x, y \in X$. The set of fixed points of $T$ is denoted by $F(T)=\{z \in X: T z=z\}$. Further, a mapping $T: X \rightarrow X$ with $F(T) \neq \emptyset$ is said to be a quasinonexpansive if $T$ satisfies $d(T x, z) \leq d(x, z)$ for any $x \in X$ and $z \in F(T)$. Moreover, $T$ is said to be $\Delta$-demiclosed if, for any bounded sequence $\left\{x_{n}\right\} \subset X$ and $x_{0} \in X$ satisfying $d\left(x_{n}, T x_{n}\right) \rightarrow 0$ and $x_{n} \Delta_{\rightarrow} x_{0}$, we have $x_{0} \in F(T)$.

\section{Tools for the main results}

In this section, we introduce some tools for using the main theorem. 
Theorem 3.1 (Kimura and Satô [7]) Let $\triangle(x, y, z)$ be a geodesic triangle in a CAT(1) space such that $d(x, y)+d(y, z)+d(z, x)<2 \pi$. Let $u=t x \oplus(1-t) y$ for some $t \in[0,1]$. Then

$$
\cos d(u, z) \sin d(x, y) \geq \cos d(x, z) \sin t d(x, y)+\cos d(y, z) \sin (1-t) d(x, y) .
$$

Corollary 3.2 (Kimura and Satô [8]) Let $\triangle(x, y, z)$ be a geodesic triangle in a CAT(1) space such that $d(x, y)+d(y, z)+d(z, x)<2 \pi$. Let $u=t x \oplus(1-t) y$ for some $t \in[0,1]$. Then

$$
\cos d(u, z) \geq t \cos d(x, z)+(1-t) \cos d(y, z) .
$$

Theorem 3.3 (Espínola and Fernández-León [9]) Let X be a complete CAT(1) space and $\left\{x_{n}\right\}$ a sequence in $X$. If $r\left(\left\{x_{n}\right\}\right)<\pi / 2$, then the following hold.

(i) $A C\left(\left\{x_{n}\right\}\right)$ consists of exactly one point;

(ii) $\left\{x_{n}\right\}$ has a $\Delta$-convergent subsequence.

Theorem 3.4 (Kimura and Satô [8]) Let $X$ be a metric space and $T$ a mapping from $X$ into itself.If $T$ is a nonexpansive with $F(T) \neq \emptyset$, then $T$ is quasinonexpansive and $\Delta$-demiclosed.

The following lemmas are important properties of real numbers and they are easy to show. Thus, we omit the proofs.

Lemma 3.5 Let $\delta$ be a real number such that $-1<\delta<0$ and $\left\{b_{n}\right\}$, $\left\{c_{n}\right\}$ real sequences satisfying $\delta \leq b_{n} \leq 1, \delta \leq c_{n} \leq 1$ and $\liminf _{n \rightarrow \infty} b_{n} c_{n} \geq 1$. Then $\lim _{n \rightarrow \infty} b_{n}=\lim _{n \rightarrow \infty} c_{n}=1$.

Lemma 3.6 Let $s \in] 0, \infty\left[\right.$ and $\left\{b_{n}\right\},\left\{c_{n}\right\}$ bounded real sequences satisfying $b_{n} \leq 0, s<c_{n}$ and $\lim _{n \rightarrow \infty} b_{n} / c_{n}=0$. Then $\lim _{n \rightarrow \infty} b_{n}=0$.

Lemma 3.7 Let $\left\{b_{n}\right\}$ and $\left\{c_{n}\right\}$ be bounded real sequences satisfying $\lim _{n \rightarrow \infty}\left(b_{n}-c_{n}\right)=0$. Then $\liminf _{n \rightarrow \infty} b_{n}=\liminf _{n \rightarrow \infty} c_{n}$.

\section{The main result}

In this section, we show the main result.

Theorem 4.1 Let $X$ be a complete CAT(1) space such that for any $u, v \in X, d(u, v)<\pi / 2$. Let $S$ and $T$ be quasinonexpansive and $\Delta$-demiclosed mappings from $X$ into itself with $F(S) \cap F(T) \neq \emptyset$. Let $\left\{\alpha_{n}\right\},\left\{\beta_{n}\right\}$ and $\left\{\gamma_{n}\right\}$ be sequences of $\left.[a, b] \subset\right] 0,1[$. Define a sequence $\left\{x_{n}\right\} \subset X$ by the following recurrence formula: $x_{1} \in X$ and

$$
\left\{\begin{array}{l}
u_{n}=\left(1-\beta_{n}\right) x_{n} \oplus \beta_{n} S x_{n}, \\
v_{n}=\left(1-\gamma_{n}\right) x_{n} \oplus \gamma_{n} T x_{n}, \\
x_{n+1}=\left(1-\alpha_{n}\right) u_{n} \oplus \alpha_{n} v_{n}
\end{array}\right.
$$

for $n \in \mathbb{N}$. Then $\left\{x_{n}\right\} \Delta$-converges to a common fixed point of $S$ and $T$. 
Proof Let $z \in F(S) \cap F(T)$. By Corollary 3.2, we have

$$
\begin{aligned}
\cos d\left(u_{n}, z\right) & \geq\left(1-\beta_{n}\right) \cos d\left(x_{n}, z\right)+\beta_{n} \cos d\left(S x_{n}, z\right) \\
& \geq\left(1-\beta_{n}\right) \cos d\left(x_{n}, z\right)+\beta_{n} \cos d\left(x_{n}, z\right) \\
& =\cos d\left(x_{n}, z\right), \\
\cos d\left(v_{n}, z\right) & \geq\left(1-\gamma_{n}\right) \cos d\left(x_{n}, z\right)+\gamma_{n} \cos d\left(T x_{n}, z\right) \\
& \geq\left(1-\gamma_{n}\right) \cos d\left(x_{n}, z\right)+\gamma_{n} \cos d\left(x_{n}, z\right) \\
& =\cos d\left(x_{n}, z\right) .
\end{aligned}
$$

Then, by Corollary 3.2 again, we have

$$
\begin{aligned}
\cos d\left(x_{n+1}, z\right) & \geq\left(1-\alpha_{n}\right) \cos d\left(u_{n}, z\right)+\alpha_{n} \cos d\left(v_{n}, z\right) \\
& \geq\left(1-\alpha_{n}\right) \cos d\left(x_{n}, z\right)+\alpha_{n} \cos d\left(x_{n}, z\right) \\
& \geq \cos d\left(x_{n}, z\right) .
\end{aligned}
$$

So, we get $d\left(x_{n+1}, z\right) \leq d\left(x_{n}, z\right)$ for all $n \in \mathbb{N}$ and there exists $d_{0}=\lim _{n \rightarrow \infty} d\left(x_{n}, z\right) \leq d\left(x_{1}, z\right)<$ $\pi / 2$.

Furthermore, by Theorem 3.1, we have

$$
\begin{aligned}
& \cos d\left(u_{n}, z\right) \sin d\left(x_{n}, S x_{n}\right) \\
& \quad \geq \cos d\left(x_{n}, z\right) \sin \left(1-\beta_{n}\right) d\left(x_{n}, S x_{n}\right)+\cos d\left(S x_{n}, z\right) \sin \beta_{n} d\left(x_{n}, S x_{n}\right) \\
& \quad \geq 2 \cos d\left(x_{n}, z\right) \sin \frac{d\left(x_{n}, S x_{n}\right)}{2} \cos \frac{\left(1-2 \beta_{n}\right) d\left(x_{n}, S x_{n}\right)}{2}
\end{aligned}
$$

and

$$
\begin{aligned}
& \cos d\left(v_{n}, z\right) \sin d\left(x_{n}, T x_{n}\right) \\
& \quad \geq \cos d\left(x_{n}, z\right) \sin \left(1-\gamma_{n}\right) d\left(x_{n}, T x_{n}\right)+\cos d\left(T x_{n}, z\right) \sin \gamma_{n} d\left(x_{n}, T x_{n}\right) \\
& \quad \geq 2 \cos d\left(x_{n}, z\right) \sin \frac{d\left(x_{n}, T x_{n}\right)}{2} \cos \frac{\left(1-2 \gamma_{n}\right) d\left(x_{n}, T x_{n}\right)}{2} .
\end{aligned}
$$

Let $d_{n}=d\left(x_{n}, z\right), s_{n}=d\left(x_{n}, S x_{n}\right) / 2$ and $t_{n}=d\left(x_{n}, T x_{n}\right) / 2$ for $n \in \mathbb{N}$. If there exists $n_{0} \in \mathbb{N}$ such that $s_{n_{0}}=t_{n_{0}}=0$, then we have $x_{n_{0}} \in F(S) \cap F(T)$ and since

$$
\begin{aligned}
x_{n_{0}+1} & =\left(1-\alpha_{n_{0}}\right)\left(\left(1-\beta_{n_{0}}\right) x_{n_{0}} \oplus \beta_{n_{0}} S x_{n_{0}}\right) \oplus \alpha_{n_{0}}\left(\left(1-\gamma_{n_{0}}\right) x_{n_{0}} \oplus \gamma_{n_{0}} T x_{n_{0}}\right) \\
& =\left(1-\alpha_{n_{0}}\right) x_{n_{0}} \oplus \alpha_{n_{0}} x_{n_{0}} \\
& =x_{n_{0}},
\end{aligned}
$$

and the proof is finished. So, we may assume $s_{n} \neq 0$ or $t_{n} \neq 0$ for all $n \in \mathbb{N}$.

If $s_{n}=0$ and $t_{n} \neq 0$, then we have $u_{n}=x_{n}$. From (2), (3), and Corollary 3.2, we get

$$
\begin{gathered}
2 \cos d_{n+1} \sin t_{n} \cos t_{n} \\
=\cos d_{n+1} \sin 2 t_{n}
\end{gathered}
$$




$$
\begin{aligned}
& \geq\left(1-\alpha_{n}\right) \cos d\left(u_{n}, z\right) \sin 2 t_{n}+\alpha_{n} \cos d\left(v_{n}, z\right) \sin 2 t_{n} \\
& \geq 2\left(1-\alpha_{n}\right) \cos d_{n} \sin t_{n} \cos t_{n}+2 \alpha_{n} \cos d_{n} \sin t_{n} \cos \left(1-2 \gamma_{n}\right) t_{n}
\end{aligned}
$$

Dividing by $2 \sin t_{n}>0$, we get

$$
\cos d_{n+1} \cos t_{n} \geq\left(1-\alpha_{n}\right) \cos d_{n} \cos t_{n}+\alpha_{n} \cos d_{n} \cos \left(1-2 \gamma_{n}\right) t_{n}
$$

If $t_{n}=0$ and $s_{n} \neq 0$, then we have $v_{n}=x_{n}$. In a similar way as above, we get

$$
\cos d_{n+1} \cos s_{n} \geq\left(1-\alpha_{n}\right) \cos d_{n} \cos \left(1-2 \beta_{n}\right) s_{n}+\alpha_{n} \cos d_{n} \cos s_{n}
$$

If $s_{n} \neq 0$ and $t_{n} \neq 0$, then from (2), (3), and Corollary 3.2, we get

$$
\begin{aligned}
& \cos d_{n+1} \sin 2 s_{n} \sin 2 t_{n} \\
& \quad \geq\left(1-\alpha_{n}\right) \cos d\left(u_{n}, z\right) \sin 2 s_{n} \sin 2 t_{n}+\alpha_{n} \cos d\left(v_{n}, z\right) \sin 2 s_{n} \sin 2 t_{n} \\
& \quad \geq 4 \cos d_{n} \sin s_{n} \sin t_{n}\left(\left(1-\alpha_{n}\right) \cos t_{n} \cos \left(1-2 \beta_{n}\right) s_{n}+\alpha_{n} \cos s_{n} \cos \left(1-2 \gamma_{n}\right) t_{n}\right) .
\end{aligned}
$$

Dividing by $4 \sin s_{n} \sin t_{n}>0$, we get

$$
\begin{aligned}
& \cos d_{n+1} \cos s_{n} \cos t_{n} \\
& \quad \geq\left(1-\alpha_{n}\right) \cos d_{n} \cos t_{n} \cos \left(1-2 \beta_{n}\right) s_{n}+\alpha_{n} \cos d_{n} \cos s_{n} \cos \left(1-2 \gamma_{n}\right) t_{n} .
\end{aligned}
$$

Therefore, (4) and (5) can be reduced to the inequality (6) and it is equivalent to

$$
\left(\frac{\varepsilon_{n} \cos s_{n}}{\alpha_{n} \cos \left(1-2 \beta_{n}\right) s_{n}}-\frac{1-\alpha_{n}}{\alpha_{n}}\right)\left(\frac{\varepsilon_{n} \cos t_{n}}{\left(1-\alpha_{n}\right) \cos \left(1-2 \gamma_{n}\right) t_{n}}-\frac{\alpha_{n}}{1-\alpha_{n}}\right) \geq 1,
$$

where $\varepsilon_{n}=\cos d_{n+1} / \cos d_{n}$ for $n \in \mathbb{N}$. It follows that $\lim _{n \rightarrow \infty} \varepsilon_{n}=\cos d_{0} / \cos d_{0}=1$. Since $\left.\left\{\alpha_{n}\right\} \subset[a, b] \subset\right] 0,1[$ for $n \in \mathbb{N}$, we get

$$
\liminf _{n \rightarrow \infty}\left(\frac{\cos s_{n}}{\alpha_{n} \cos \left(1-2 \beta_{n}\right) s_{n}}-\frac{1-\alpha_{n}}{\alpha_{n}}\right)\left(\frac{\cos t_{n}}{\left(1-\alpha_{n}\right) \cos \left(1-2 \gamma_{n}\right) t_{n}}-\frac{\alpha_{n}}{1-\alpha_{n}}\right) \geq 1 .
$$

Then we show that there exists $n_{0} \in \mathbb{N}$ such that for all $n \geq n_{0}$, the following hold:

$$
-\frac{1}{2} \leq \frac{\cos s_{n}}{\alpha_{n} \cos \left(1-2 \beta_{n}\right) s_{n}}-\frac{1-\alpha_{n}}{\alpha_{n}} \leq 1
$$

and

$$
-\frac{1}{2} \leq \frac{\cos t_{n}}{\left(1-\alpha_{n}\right) \cos \left(1-2 \gamma_{n}\right) t_{n}}-\frac{\alpha_{n}}{1-\alpha_{n}} \leq 1
$$

First, we show the right inequality of (8). Since $\left.\left\{\beta_{n}\right\} \subset[a, b] \subset\right] 0,1[$ for $n \in \mathbb{N}$, we get $\cos s_{n} \leq \cos \left|1-2 \beta_{n}\right| s_{n}=\cos \left(1-2 \beta_{n}\right) s_{n}$. Hence we get

$$
\frac{\cos s_{n}}{\alpha_{n} \cos \left(1-2 \beta_{n}\right) s_{n}}-\frac{1-\alpha_{n}}{\alpha_{n}} \leq \frac{1}{\alpha_{n}}-\frac{1-\alpha_{n}}{\alpha_{n}}=1
$$


By the same method as above, the right inequality of (9) also holds. Next, let us show the left inequality of (8). If it does not hold, then letting

$$
\sigma_{n}=\frac{\cos s_{n}}{\alpha_{n} \cos \left(1-2 \beta_{n}\right) s_{n}}-\frac{1-\alpha_{n}}{\alpha_{n}} \quad \text { and } \quad \tau_{n}=\frac{\cos t_{n}}{\left(1-\alpha_{n}\right) \cos \left(1-2 \gamma_{n}\right) t_{n}}-\frac{\alpha_{n}}{1-\alpha_{n}},
$$

we can find a subsequence $\left\{\sigma_{n_{i}}\right\} \subset\left\{\sigma_{n}\right\}$ such that $\sigma_{n_{i}}<-1 / 2$ for $i \in \mathbb{N}$ and $\lim _{i \rightarrow \infty} \sigma_{n_{i}}=$ $\sigma \leq-1 / 2$. Since $\left.\left\{\alpha_{n}\right\},\left\{\gamma_{n}\right\} \subset[a, b] \subset\right] 0,1\left[\right.$ and $\left\{t_{n}\right\} \subset\left[0, \pi / 4\left[\subset\left[0, \pi / 2\left[\right.\right.\right.\right.$, we have $\left\{\tau_{n}\right\}$ is bounded. Therefore, by taking a subsequence again if necessary, we may assume that $\left\{\tau_{n_{i}}\right\}$ converges to $\tau \in \mathbb{R}$. Then, by (7), we get $\sigma \tau=\lim _{i \rightarrow \infty} \sigma_{n_{i}} \tau_{n_{i}} \geq \liminf _{n \rightarrow \infty} \sigma_{n} \tau_{n} \geq 1$. Hence we may assume that $\tau_{n_{i}}<0$ for all $i \in \mathbb{N}$. Since $\sqrt{2} / 2<\cos s_{n}, \sqrt{2} / 2<\cos t_{n}, 0<\cos (1-$ $\left.2 \beta_{n}\right) s_{n} \leq 1,0<\cos \left(1-2 \gamma_{n}\right) t_{n} \leq 1$ and $\left.\left\{\alpha_{n}\right\} \subset[a, b] \subset\right] 0,1[$, we also have

$$
0<\frac{\sqrt{2}}{2 b} \leq \frac{\cos s_{n}}{\alpha_{n} \cos \left(1-2 \beta_{n}\right) s_{n}} \quad \text { and } \quad 0<\frac{\sqrt{2}}{2(1-a)} \leq \frac{\cos t_{n}}{\left(1-\alpha_{n}\right) \cos \left(1-2 \gamma_{n}\right) t_{n}} .
$$

Let $\rho$ be a real number such that

$$
0<\rho<\min \left\{\frac{\sqrt{2}}{2 b}, \frac{\sqrt{2}}{2(1-a)}, \frac{1-b}{b}+\frac{a}{1-a}\right\} .
$$

Then, by (10), we get

$$
\rho-\frac{1-\alpha_{n_{i}}}{\alpha_{n_{i}}} \leq \sigma_{n_{i}}<0 \quad \text { and } \quad \rho-\frac{\alpha_{n_{i}}}{1-\alpha_{n_{i}}} \leq \tau_{n_{i}}<0 .
$$

Then, by (11) and (12), we have

$$
\begin{aligned}
\sigma_{n_{i}} \tau_{n_{i}} & \leq\left(\rho-\frac{1-\alpha_{n_{i}}}{\alpha_{n_{i}}}\right)\left(\rho-\frac{\alpha_{n_{i}}}{1-\alpha_{n_{i}}}\right) \\
& =\rho^{2}-\left(\frac{1-\alpha_{n_{i}}}{\alpha_{n_{i}}}+\frac{\alpha_{n_{i}}}{1-\alpha_{n_{i}}}\right) \rho+1 \\
& \leq \rho^{2}-\left(\frac{1-b}{b}+\frac{a}{1-a}\right) \rho+1 \\
& =\rho\left(\rho-\left(\frac{1-b}{b}+\frac{a}{1-a}\right)\right)+1 .
\end{aligned}
$$

Thus, as $i \rightarrow \infty$, we have

$$
1 \leq \sigma \tau \leq \rho\left(\rho-\left(\frac{1-b}{b}+\frac{a}{1-a}\right)\right)+1<1 .
$$

This is a contradiction. We also obtain the left inequality of (9) in a similar way. Hence we get

$$
\lim _{n \rightarrow \infty}\left(\frac{\cos s_{n}}{\alpha_{n} \cos \left(1-2 \beta_{n}\right) s_{n}}-\frac{1-\alpha_{n}}{\alpha_{n}}\right)=\lim _{n \rightarrow \infty}\left(\frac{\cos t_{n}}{\left(1-\alpha_{n}\right) \cos \left(1-2 \gamma_{n}\right) t_{n}}-\frac{\alpha_{n}}{1-\alpha_{n}}\right)=1
$$

by Lemma 3.5, (8), and (9). Furthermore, from (14), we get

$$
\lim _{n \rightarrow \infty} \frac{\cos s_{n}-\cos \left(1-2 \beta_{n}\right) s_{n}}{\alpha_{n} \cos \left(1-2 \beta_{n}\right) s_{n}}=0 .
$$


By Lemma 3.6 and (15), we get

$$
\lim _{n \rightarrow \infty}\left(\cos s_{n}-\cos \left(1-2 \beta_{n}\right) s_{n}\right)=0
$$

Moreover, by Lemma 3.7 and (16), we get

$$
\liminf _{n \rightarrow \infty} \cos s_{n}=\liminf _{n \rightarrow \infty} \cos \left(1-2 \beta_{n}\right) s_{n}=\liminf _{n \rightarrow \infty} \cos \left|1-2 \beta_{n}\right| s_{n} .
$$

Hence we get

$$
\limsup _{n \rightarrow \infty} s_{n}=\limsup _{n \rightarrow \infty}\left(\left|1-2 \beta_{n}\right| s_{n}\right) \leq \limsup _{n \rightarrow \infty}\left|1-2 \beta_{n}\right| \limsup _{n \rightarrow \infty} s_{n},
$$

and we have

$$
0 \geq\left(1-\limsup _{n \rightarrow \infty}\left|1-2 \beta_{n}\right|\right) \limsup _{n \rightarrow \infty} s_{n}=\liminf _{n \rightarrow \infty}\left(1-\left|1-2 \beta_{n}\right|\right) \limsup _{n \rightarrow \infty} s_{n} .
$$

Since $\left.\left\{\beta_{n}\right\} \subset[a, b] \subset\right] 0,1\left[\right.$ for $n \in \mathbb{N}$, we have $\liminf _{n \rightarrow \infty}\left(1-\left|1-2 \beta_{n}\right|\right)>0$ and thus, $\limsup \operatorname{sum}_{n \rightarrow \infty} s_{n} \leq 0$. Therefore, we get $\limsup _{n \rightarrow \infty} s_{n}=0$ and we also get $\limsup _{n \rightarrow \infty} t_{n}=0$. It implies $d\left(x_{n}, S x_{n}\right) \rightarrow 0$ and $d\left(x_{n}, T x_{n}\right) \rightarrow 0$.

Next, let $\left\{y_{k}\right\}$ be a subsequence of $\left\{x_{n}\right\}$. Since $r\left(\left\{x_{n}\right\}\right) \leq d_{0}<\pi / 2$, by Theorem 3.3(i), there exists a unique asymptotic center $x_{0}$ of $\left\{y_{k}\right\}$. Moreover, since $r\left(\left\{y_{k}\right\}\right)<\pi / 2$, by Theorem 3.3(ii), there exists a subsequence $\left\{z_{l}\right\}$ of $\left\{y_{k}\right\}$ such that $z_{l} \stackrel{\Delta}{\rightarrow} z_{0} \in X$. Further, since $d\left(z_{l}, S z_{l}\right) \rightarrow 0, d\left(z_{l}, T z_{l}\right) \rightarrow 0$ and $S, T$ are $\Delta$-demiclosed, we have $z_{0} \in F(S) \cap F(T)$. Then we can show that $x_{0}=z_{0}$, i.e., $x_{0} \in F(S) \cap F(T)$. If not, from the uniqueness of the asymptotic centers $x_{0}, z_{0}$ of $\left\{y_{k}\right\},\left\{z_{l}\right\}$, respectively, due to Theorem 3.3(i), we have

$$
\begin{aligned}
\limsup _{k \rightarrow \infty} d\left(y_{k}, x_{0}\right) & <\limsup _{k \rightarrow \infty} d\left(y_{k}, z_{0}\right) \\
& =\lim _{n \rightarrow \infty} d\left(x_{n}, z_{0}\right) \\
& =\limsup _{l \rightarrow \infty} d\left(z_{l}, z_{0}\right) \\
& <\limsup _{l \rightarrow \infty} d\left(z_{l}, x_{0}\right) \\
& \leq \limsup _{k \rightarrow \infty} d\left(y_{k}, x_{0}\right) .
\end{aligned}
$$

This is a contradiction. Hence we get $x_{0} \in F(S) \cap F(T)$. Next, we show that for any subsequences of $\left\{x_{n}\right\}$, their asymptotic center consists of the unique element. Let $\left\{u_{k}\right\},\left\{v_{k}\right\}$ be subsequences of $\left\{x_{n}\right\}, x_{0} \in A C\left(\left\{u_{k}\right\}\right)$ and $x_{0}^{\prime} \in A C\left(\left\{v_{k}\right\}\right)$. We show $x_{0}=x_{0}^{\prime}$ by using contradiction. Assume $x_{0} \neq x_{0}^{\prime}$. Then $x_{0}^{\prime} \notin A C\left(\left\{u_{k}\right\}\right)$ and $x_{0} \notin A C\left(\left\{v_{k}\right\}\right)$ by Theorem 3.3(i). It follows that

$$
\begin{array}{r}
\limsup _{k \rightarrow \infty} d\left(u_{k}, x_{0}\right)<\limsup _{k \rightarrow \infty} d\left(u_{k}, x_{0}^{\prime}\right) \\
=\lim _{n \rightarrow \infty} d\left(x_{n}, x_{0}^{\prime}\right) \\
=\limsup _{k \rightarrow \infty} d\left(v_{k}, x_{0}^{\prime}\right)
\end{array}
$$




$$
\begin{aligned}
& <\limsup _{k \rightarrow \infty} d\left(v_{k}, x_{0}\right) \\
& =\lim _{n \rightarrow \infty} d\left(x_{n}, x_{0}\right) \\
& =\limsup _{k \rightarrow \infty} d\left(u_{k}, x_{0}\right) .
\end{aligned}
$$

This is a contradiction. Hence we get $x_{0}=x_{0}^{\prime}$. Therefore, we have $\left\{x_{n}\right\} \Delta$-converges to a common fixed point of $S$ and $T$.

By Theorem 3.4, we know that a nonexpansive mapping having a fixed point satisfies the assumptions in Theorem 4.1. Thus, we get the following result.

Corollary 4.2 Let $X$ be a complete CAT(1) space such that for any $u, v \in X, d(u, v)<\pi / 2$. Let $S$ and $T$ be nonexpansive mappings of $X$ into itself such that $F(S) \cap F(T) \neq \emptyset$. Let $\left\{\alpha_{n}\right\}$, $\left\{\beta_{n}\right\}$ and $\left\{\gamma_{n}\right\}$ be sequences in $\left.[a, b] \subset\right] 0,1\left[\right.$. Define a sequence $\left\{x_{n}\right\} \subset X$ as the following recurrence formula: $x_{1} \in X$ and

$$
\left\{\begin{array}{l}
u_{n}=\left(1-\beta_{n}\right) x_{n} \oplus \beta_{n} S x_{n}, \\
v_{n}=\left(1-\gamma_{n}\right) x_{n} \oplus \gamma_{n} T x_{n} \\
x_{n+1}=\left(1-\alpha_{n}\right) u_{n} \oplus \alpha_{n} v_{n}
\end{array}\right.
$$

for $n \in \mathbb{N}$. Then $\left\{x_{n}\right\} \Delta$-converges to a common fixed point of $S$ and $T$.

\section{An application to the image recovery}

The image recovery problem is formulated as to find the nearest point in the intersection of family of closed convex subsets from a given point by using corresponding metric projection of each subset. In this section, we consider this problem for two subsets of a complete CAT(1) space.

Theorem 5.1 Let $X$ be a complete CAT(1) space such that for any $u, v \in X, d(u, v)<\pi / 2$. Let $C_{1}$ and $C_{2}$ be nonempty closed convex subsets of $X$ such that $C_{1} \cap C_{2} \neq \emptyset$. Let $P_{1}$ and $P_{2}$ be metric projections onto $C_{1}$ and $C_{2}$, respectively. Let $\left\{\alpha_{n}\right\},\left\{\beta_{n}\right\}$ and $\left\{\gamma_{n}\right\}$ be real sequences in $[a, b] \subset] 0,1\left[\right.$. Define a sequence $\left\{x_{n}\right\} \subset X$ by the following recurrence formula: $x_{1} \in X$ and

$$
\left\{\begin{array}{l}
u_{n}=\left(1-\beta_{n}\right) x_{n} \oplus \beta_{n} P_{1} x_{n} \\
v_{n}=\left(1-\gamma_{n}\right) x_{n} \oplus \gamma_{n} P_{2} x_{n} \\
x_{n+1}=\left(1-\alpha_{n}\right) u_{n} \oplus \alpha_{n} v_{n}
\end{array}\right.
$$

for $n \in \mathbb{N}$. Then $\left\{x_{n}\right\} \Delta$-converges to a fixed point of the intersection of $C_{1}$ and $C_{2}$.

Proof We see that $P_{1}$ and $P_{2}$ are quasinonexpansive [9] and $\Delta$-demiclosed [8]. Further, we also get $F\left(P_{1}\right)=C_{1}$ and $F\left(P_{2}\right)=C_{2}$. Thus, letting $S=P_{1}$ and $T=P_{2}$ in Theorem 4.1, we obtain the desired result. 
Authors' contributions

The authors have contributed in this work on an equal basis. All authors read and approved the final manuscript.

\section{Acknowledgements}

The authors thank the anonymous referees for their valuable comments and suggestions. The first author is supported by Grant-in-Aid for Scientific Research No. 22540175 from the Japan Society for the Promotion of Science.

Received: 15 October 2013 Accepted: 23 January 2014 Published: 13 Feb 2014

\section{References}

1. Mann, WR: Mean value methods in iteration. Proc. Am. Math. Soc. 4, 506-510 (1953)

2. Reich, S: Weak convergence theorems for nonexpansive mappings in Banach spaces. J. Math. Anal. Appl. 67, 274-276 (1979)

3. Takahashi, W, Tamura, T: Convergence theorems for nonexpansive mappings in Banach spaces. J. Math. Anal. Appl. 67(1), 45-56 (1998)

4. Dhompongsa, S, Panyanak, B: On $\Delta$-convergence theorems in CAT(0) spaces. Comput. Math. Appl. 56(10), 2572-2579 (2008)

5. Kimura, Y, Saejung, S, Yotkaew, P: The Mann algorithm in a complete geodesic space with curvature bounded above. Fixed Point Theory Appl. 2013, 1-13 (2013)

6. Bridson, MR, Haefliger, A: Metric Spaces of Non-Positive Curvature. Grundlehren der Mathematischen Wissenschaften, vol. 319. Springer, Berlin (1999)

7. Kimura, Y, Satô, K: Convergence of subsets of a complete geodesic space with curvature bounded above. Nonlinear Anal. 75(13), 5079-5085 (2012)

8. Kimura, Y, Satô, K: Halpern iteration for strongly quasinonexpansive mappings on a geodesic space with curvature bounded above by one. Fixed Point Theory Appl. 2013, Article ID 7 (2013)

9. Espínola, R, Fernández-León, A: CAT( $\kappa)$-spaces, weak convergence and fixed points. J. Math. Anal. Appl. 353(1), 410-427 (2009)

10.1186/1029-242X-2014-72

Cite this article as: Kimura and Nakagawa: Another type of Mann iterative scheme for two mappings in a complete geodesic space. Journal of Inequalities and Applications 2014, 2014:72

\section{Submit your manuscript to a SpringerOpen ${ }^{\circ}$ journal and benefit from:}

- Convenient online submission

- Rigorous peer review

Immediate publication on acceptance

Open access: articles freely available online

- High visibility within the field

- Retaining the copyright to your article 